\title{
Philosophiques
}

\section{Introduction au dossier - Usages de la réflexivité en philosophie allemande}

Augustin Dumont

Volume 43, numéro 2, automne 2016

Dossier. Usages de la réflexivité en philosophie allemande

URI : https://id.erudit.org/iderudit/1038202ar

DOI : https://doi.org/10.7202/1038202ar

Aller au sommaire du numéro

Éditeur(s)

Société de philosophie du Québec

ISSN

0316-2923 (imprimé)

1492-1391 (numérique)

Découvrir la revue

Citer ce document

Dumont, A. (2016). Introduction au dossier - Usages de la réflexivité en philosophie allemande. Philosophiques, 43(2), 183-184.

https://doi.org/10.7202/1038202ar d'utilisation que vous pouvez consulter en ligne.

https://apropos.erudit.org/fr/usagers/politique-dutilisation/ 


\title{
Introduction au dossier - Usages de la réflexivité en philosophie allemande
}

\author{
AUGUSTIN DUMONT \\ Université de Montréal
}

La notion de réflexivité a été investie de manière persistante aussi bien par ladite philosophie classique allemande (klassische deutsche Philosophie), qui court de Kant à l'aube du marxisme, que par la philosophie allemande contemporaine (des écoles néokantiennes à la dernière École de Francfort en passant par la phénoménologie). Décliné de différentes manières (Reflexivität, Nachdenken, Überlegung, etc.) selon le contexte argumentatif, un tel concept a ceci de particulier qu'il n'est jamais mobilisé, dans ces traditions, comme simple adjuvant au raisonnement. En effet, il est bien plutôt indicatif de ce que la philosophie allemande voit dans le retour sur soi de la pensée le cœur de son héritage de la modernité. Il ne s'agit pas tant, dans ce cadre, d'ériger la réflexivité en garante d'une position de surplomb que le sujet serait supposé avoir vis-à-vis de ses propres représentations du monde, comme on le croit parfois, que d'en faire le vecteur d'une libération du sujet. Loin de confiner à la dimension illusoire de maîtrise ou de contrôle de son monde par le sujet, le geste réflexif signale qu'il n'y a pas de retour sur soi qui ne serait ipso facto invitation à penser par soi-même, et ainsi à libérer la raison de toute hétéronomie.

Le présent numéro de Philosophiques entend proposer à ses lectrices et lecteurs quelques variations libres sur ce que le recours à la réflexivité a pu rendre possible dans la pensée allemande. De la conception kantienne de la Critique (sur laquelle revient Charlotte Sabourin) à l'idée d'une clarification (Klärung) réflexive chez Adorno (sur laquelle se penche Franck Fischbach), la philosophie allemande classique et contemporaine se garde de voir dans la réflexion le retour tardif, voire optionnel, d'une pensée fatiguée sur ses propres opérations dans le sens. S'il en est ainsi, c'est parce que l'Aufklärung demeure, par-delà toutes les critiques dont elle a pu faire l'objet chez eux, le point de référence des philosophes allemands de la réflexivité. Le projet moderne d'une émancipation de l'argument d'autorité et des tutelles traditionnelles qui s'exercent sur la pensée passe pour ainsi dire de lui-même à la puissance à l'époque des Lumières. La raison éclairée est celle dont l'essence consiste précisément à faire retour sur soi, non pas seulement dans l'aprèscoup, mais afin d'engager ici et maintenant un rapport au monde qui soit un rapport à la fois critique et créateur de possibles. Chez Fichte, l'agir en général, dont la connaissance (l'acte de connaître) devient une modalité, se définit précisément comme pure et absolue autoréflexivité. C'est là la source de toute légitimation de la connaissance comme aussi de sa possibilisation 
(ce point de vue architectonique est ici médité par Alexander Schnell). Il convient de ne lire en un tel projet nulle consécration d'une pensée autarcique, complaisamment repliée sur elle-même, tant la réflexion a intrinsèquement à voir avec le problème de l'altérité (ce que montre Augustin Dumont). La pensée fichtéenne en impose, au fond, pour une véritable systématique des points de vue, laquelle s'appuie de la manière la plus féconde sur les concepts de vie et d'absolu (ce que montre Quentin Landenne). De manière générale, la réflexivité apparaît comme un opérateur central dans la constitution d'une pensée dont l'horizon est toujours le monde commun et les conditions du vivre-ensemble (ce qu'Olivier Tinland met en évidence dans le «kantisme» de Habermas). 